\title{
A method to quickly predict dispersion for impact point of high spin rate projectile
}

\author{
Xiao-lin Luo \\ Science and Technology on Electormechanical Dynamic Control Laboratory, Beijing Institute of \\ Technology, Beijing 100081, China \\ (ouwenlxl@163.com)
}

\begin{abstract}
Keywords: Precision Error, Mean Point of Impact Error, Monte Carlo analysis, The Root Mean Square Method.

Abstract. A method for real time in flight prediction of the ground impact point and dispersion of indirect fire projectiles is investigated. A three degree of freedom projectile model solution is used to be determined the total dispersion of trajectory. With the root mean square method, mean point of impact error (MPI) of projectile is simulation. the final simulation verifies that the method proposed is use for determined the total dispersion of trajectory.
\end{abstract}

\section{Introduction}

Dispersion is a measure for the deviation of the projectile's trajectory from the trajectory of some standard projectile, called nominal trajectory. Simulation of the impact point of unguided projectile has as input data characteristics of the projectile, atmospheric conditions and launching conditions. In reality all these input data may have some variations around a nominal value that implies a variation in trajectory path. Therefore, there is always an area of possible impact points. The only way to minimize this impact area is to impose some guided projectile. restrictions in manufacturing tolerances, since no restrictions can be made in minimizing the errors of atmospheric conditions. The aim of this paper is to determine the total dispersion of trajectory and to estimate the impact point using the quickly predicted method. ${ }^{[1]}$

There are some methods to estimate the dispersion of impact point for a projectile ${ }^{[2]}$ :

The Root Mean Square Method simulates the projectile impact point perturbing one parameter at time and the results are compared with the nominal results. The sum of squares deviations for all parameters is square of total deviation.

Monte Carlo method of dispersion removes smaller dispersion parameters. Each input parameter is selected randomly in the defined ranges and used in the simulation of trajectory.

The Method of covariance matrix: In probability theory and statistics, covariance is a measure of how much two variables change together. A covariance matrix is a matrix whose element in the $\mathrm{i}, \mathrm{j}$ position is the covariance between the $i$ th and $j$ th elements of a random vector (that is, of a vector of random variables). Each element of the vector is a scalar random variable, either with a finite number of observed empirical values or with a finite or infinite number of potential values specified by a theoretical joint probability distribution of all the random variables ${ }^{[3]}$.

Monte Carlo analysis may require several hundreds to thousands of simulation runs. In order to quickly predicted dispersion for impact point of high spin rate projectile. we choose the Root Mean Square Method.

In this paper, the 3 DOF model is used to calculate nominal trajectory firstly. Then the model of Mean point of impact error (MPI) of projectile to be determined the total dispersion of trajectory. Finally some deviation are obtained to the major contributor to the accuracy results. 


\section{SYSTEM ERROR MODEL}

\section{A 3 DOF model}

The trajectory of a projectile can be modeled using different methodologies. The common methodologies are the point mass model, and six degree of model. The six degree of model is capable of predicting the trajectory with good accuracies, but requires more computing resources and time ${ }^{[4]}$.

The total drag force with the addition of the crosswind acting on the projectile is given by

$$
\mathrm{F}_{\mathrm{D}}=\frac{1}{2} \mathrm{~S}_{\mathrm{ref}} \rho v_{\mathrm{r}}^{2} \mathrm{C}_{\mathrm{D}}
$$

Consequently, the drag force can be resolved into two components, the range and deflection components are given by

$$
\begin{gathered}
\mathrm{F}_{\mathrm{DR}}=\mathrm{F}_{\mathrm{D}} \cos \varphi \\
\mathrm{F}_{\mathrm{DD}}=\mathrm{F}_{\mathrm{D}} \sin \varphi
\end{gathered}
$$

where FDR: Drag force in the range direction,

FDD: Drag force in the deflection direction,

$\varphi$ : Angle between the projectile axis and trajectory

The range component of the drag force, FDR is used to calculate the acceleration components in the range direction and the deflection component is used to calculate the acceleration due to the crosswind. Thus the horizontal and vertical components of the acceleration on the projectile in range can be derived as

$$
\begin{aligned}
& \mathrm{a}_{\mathrm{x}}=\frac{-\mathrm{F}_{\mathrm{DR}} \cos \theta}{\mathrm{m}} \\
& \mathrm{a}_{\mathrm{y}}=-\mathrm{g}-\frac{-\mathrm{F}_{\mathrm{DR}} \sin \theta}{\mathrm{m}}
\end{aligned}
$$

The acceleration of the projectile in the deflection direction is given by

$$
\mathrm{a}_{\mathrm{DW}}=\frac{\mathrm{F}_{\mathrm{DD}}}{\mathrm{m}}
$$

Therefore, the drift due to the wind effect can be written as

$$
\mathrm{Z}_{\mathrm{w}}=\frac{1}{2} \mathrm{a}_{\mathrm{Dw}} \mathrm{t}^{2}
$$

The drift due to rotating projectile effects can be written as

$$
\begin{aligned}
& \mathrm{Z}_{\mathrm{p}}=\mathrm{k}^{\mathrm{w}} \mathrm{t}^{2} \\
& \mathrm{Z}=\mathrm{Z}_{\mathrm{w}}+\mathrm{Z}_{\mathrm{p}}
\end{aligned}
$$

The velocity of the projectile can be evaluated by integrating the acceleration:

$\mathrm{a}=\frac{\mathrm{dv}}{\mathrm{dt}}=\frac{\mathrm{v}(\mathrm{t}+\mathrm{dt})-\mathrm{v}(\mathrm{t})}{\mathrm{dt}}$

Therefore, for a given time step dt, the horizontal and vertical component of the velocity can be evaluated by

$$
\begin{aligned}
& v_{x}(t+d t)=a_{x} d t+v_{x}(t) \\
& v_{y}(t+d t)=a_{y} d t+v_{y}(t)
\end{aligned}
$$

Similarly from the velocity equations, the horizontal and vertical component of the displacement can be derived as

$$
\begin{aligned}
& x(t+d t)=v_{x} d t+x(t) \\
& y(t+d t)=v_{y} d t+y(t)
\end{aligned}
$$

where $\mathrm{x}$ :Displacement in the range direction, y: Displacement in the vertical direction. 


\section{B. Mean point of impact error model (MPI) of projectile}

The MPI error is associated with the occasion-to-occasion variation about the target that is affected by the aiming error. In addition, the time and meteorological conditions difference affects the MPI error. The range and deflection MPI errors are given by,

$$
\begin{gathered}
\sigma_{\mathrm{MPI}, \mathrm{X}}{ }^{2}=\left[\frac{\partial \mathrm{x}}{\partial \mathrm{p}}\right]^{2}\left(\sigma_{\mathrm{p}}^{2}+\sigma_{\mathrm{D}}^{2}\right)+\left(\frac{\partial \mathrm{x}}{\partial \mathrm{T}} \sigma_{\mathrm{T}}\right)^{2}+\left(\frac{\partial \mathrm{x}}{\partial \mathrm{w}} \sigma_{\mathrm{w}}\right)^{2}+\left(\frac{\partial \mathrm{x}}{\partial \mathrm{v}} \sigma_{\mathrm{v}}\right)^{2}+\left(\frac{\partial \mathrm{x}}{\partial \theta} \sigma_{\mathrm{AIM}-\mathrm{EL}}\right)^{2}+\sigma_{\mathrm{LOC}-\mathrm{x}}{ }^{2} \\
\sigma_{\mathrm{MPI}, \mathrm{Z}}=\left(\frac{\partial \mathrm{Z}}{\partial \mathrm{w}} \sigma_{\mathrm{w}}\right)^{2}+\left(\frac{\partial \mathrm{Z}}{\partial \mathrm{L}} \sigma_{\mathrm{L}}\right)^{2}+\left(\frac{\partial \mathrm{Z}}{\partial \alpha} \sigma_{\mathrm{AIM}-\mathrm{AZ}}\right)^{2}+\sigma_{\mathrm{LOC}-\mathrm{z}^{2}}
\end{gathered}
$$

Where

$\sigma_{\mathrm{MPIX}}$ MPI error in range ,

$\sigma_{\mathrm{MPI}, \mathrm{Z}} \quad$ MPI error in deflection

$\sigma_{\mathrm{T}} \quad$ Error budget in temperature

$\sigma_{\mathrm{w}} \quad$ Error budget in wind speed

$\sigma_{\text {AIM-EL }}$ Error budget in aiming error for QE

$\sigma_{\text {AIM-Az }}$ Error budget in aiming error for azimuth

$\sigma_{\text {LOC }-\mathrm{x}}$ Error budget in location accuracy for range

$\sigma_{\mathrm{LOC}-\mathrm{z}}$ Error budget in location accuracy for deflection

$\begin{array}{llcc}\sigma_{\mathrm{L}} & \text { Error budget in lift } & \sigma_{\rho} & \text { Error budget in density } \\ \frac{\partial \mathrm{X}}{\partial \rho} & \text { Density unit effect, } & \frac{\partial \mathrm{X}}{\partial \mathrm{T}} & \text { Temperature unit effect, } \\ \frac{\partial \mathrm{X}}{\partial \mathrm{w}} & \text { Range wind unit effect, } & \frac{\partial \mathrm{z}}{\partial \mathrm{w}} & \text { Cross wind unit effect, } \\ \frac{\partial \mathrm{Z}}{\partial \mathrm{L}} & \text { Lift unit effect, } & \frac{\partial \mathrm{Z}}{\partial \mathrm{c}} & \text { Azimuth unit effect. }\end{array}$

\section{Unit Effects in MPI Error}

Since a unit effect measures the change in the range of deflection due to a change in the firing condition, the Density Unit Effect, Muzzle Velocity Unit Effect ${ }^{[5]}$.

The Temperature Unit Effect is calculated by varying the temperature by 10 percent above the temperature in standard condition. The temperature unit effect is given as

$$
\begin{aligned}
& \frac{d X}{d T}=\frac{X_{2}-X_{1}}{T_{2}-T_{1}} \\
& T_{1}=T_{0} \\
& T_{2}=1.1 \times T_{0}
\end{aligned}
$$

The ranges, $\mathrm{X} 2$ and $\mathrm{X} 1$ are calculated with the launch elevations of $\mathrm{T} 2$ and $\mathrm{T} 1$ respectively, and keeping all other variables and inputs constant. Similarly, Other Unit Effects in MPI Error is calculated .

\section{SIMULATION AND ANALYSIS}

Under the circumstances that no atmospheric disturbances were present, no manufacturing, reading or measuring errors were made, the nominal trajectory have been generated[6]. Table 1 present the coefficients and some initial parameter for the typical $155 \mathrm{~mm}$ projectile. The Density $(\sigma \rho)$, Temperature $(\sigma \mathrm{T})$ and Wind Error Budgets $(\sigma \mathrm{W})$ are the variation of the meteorological conditions from the standard atmosphere.

Table 1 some initial parameter and the coefficients of the typical $155 \mathrm{~mm}$ projectile

\begin{tabular}{|c|c|c|c|c|c|c|c|c|c|}
\hline $\begin{array}{c}\text { Projectil } \\
\text { e Mass }\end{array}$ & $\begin{array}{c}\text { Muzzle } \\
\text { Velocity }\end{array}$ & $\begin{array}{c}\text { Deflection } \\
\text { acceleration }\end{array}$ & $\sigma_{v}$ & $\sigma_{\mathrm{D}}$ & $\sigma_{\mathrm{L}}$ & $\begin{array}{r}\sigma_{\mathrm{AMM}} \\
-\mathrm{EL}\end{array}$ & $\begin{array}{r}\sigma_{\mathrm{AIM}} \\
-\mathrm{AZ}\end{array}$ & $\sigma_{\mathrm{LOC}-\mathrm{X}}$ & $\sigma_{\mathrm{LOC}-\mathrm{Z}}$ \\
\hline $42 \mathrm{~kg}$ & $684.5 \mathrm{~m} / \mathrm{s}$ & $0.2029 \mathrm{~m} / \mathrm{s}^{\wedge} 2$ & $3 \mathrm{~m} / \mathrm{s}$ & $1 \%$ & $1 \%$ & 0.5 & 4 & $15 \mathrm{~m}$ & $15 \mathrm{~m}$ \\
\hline
\end{tabular}


In order to investigate the trajectory parameters of the given unguided projectile, three cases will be chosen corresponding to the desirered range: $5000 \mathrm{~m}, 10000 \mathrm{~m}, 15000 \mathrm{~m}$. These cases will give us the possibility to compare the results corresponding to the desirered range .

To investigate the individual influence on trajectory, every parameter will be modified inside the proper variation interval. Table 2 presents the results of the impact point distance error and some major parameters effect to error of range and deflection.

Table 2the impact point distance error and some major parameters effect to error of range and deflection

\begin{tabular}{|c|c|c|c|c|c|c|c|c|c|c|}
\hline range & $\sigma_{M P I, X}$ & $\sigma_{M P I, Z}$ & $\frac{\partial X}{\partial W}$ & $\frac{\partial X}{\partial \theta}$ & $\frac{\partial X}{\partial v}$ & $\frac{\partial X}{\partial \rho}$ & $\frac{\partial X}{\partial T}$ & $\frac{\partial Z}{\partial w}$ & $\frac{\partial Z}{\partial L}$ & $\frac{\partial Z}{\partial \alpha}$ \\
\hline 5000 & 49 & 34 & 3.6 & -651 & 8.9 & -26.7 & 3 & 8.6 & 9.3 & 5 \\
\hline 10000 & 93 & 62 & 13.6 & -548 & 17.4 & -53 & 6.4 & 17.5 & 8.7 & 9.8 \\
\hline 15000 & 145.8 & 92 & 31 & -338 & 25 & -78 & 11.4 & 27 & 7.4 & 14.8 \\
\hline
\end{tabular}

In MPI error for range, the major contributors to the accuracy results are the muzzle velocity and the range wind. In the MPI error for deflection, the major contributors to the accuracy result are the cross wind effect and the gun aiming in the azimuth plane.

\section{CONCLUSION}

After model building of Mean point of impact error model (MPI) of projectile, A 3 DOF model is sufficient to show the general behavior of the trajectory of an artillery-fired projectile. With the root mean square method, mean point of impact error (MPI) of projectile is simulation. the final simulation verifies that the method proposed is use for determined the total dispersion of trajectory. The major contributing factors to dispersion of unguided artillery projectiles are deviations from nominal values in muzzle velocity and meteorological conditions (wind, air density, temperature), aiming errors of the gun and deviation in projectile properties.

\section{References:}

[1] Jeffrey A. Isaacson, David R. Vaughan,"Estimation and Prediction of Ballistic Missile Trajectories", RAND, 1996.

[2] Cristina Mihailescu. "The Analysis of Dispersion for Trajectories of Fire-extinguishing Rocket", Recent Advances in Fluid Mechanics and Heat \& Mass Transfer,2004

[4] Driels, Morris R., "Weaponeering: Conventional Weapon System Effectiveness," Reston, Virginia, American Institute of Aeronautics and Astronautics, 2004.

[5] Fann, Chee Meng . Development of an artillery accuracy model. Naval Postgraduate School 2006

[6] Backman, Marvin E., "Terminal Ballistics," China Lake, California, Naval Weapons Center, 1976. 